\title{
The in vitro activity of fatty diamines and amino alcohols against mixed amastigote and trypomastigote Trypanosoma cruzi forms
}

\author{
Policarpo Ademar Sales Júnior ${ }^{1}$, Celso Oliveira Rezende Júnior², \\ Mireille Le Hyaric ${ }^{2}$, Mauro Vieira de Almeida ${ }^{2}{ }^{+}$, Alvaro José Romanha ${ }^{1,3}$ \\ 'Laboratório de Parasitologia Celular e Molecular, Centro de Pesquisas René Rachou-Fiocruz, Belo Horizonte, MG, Brasil \\ ${ }^{2}$ Departamento de Química, Universidade Federal de Juiz de Fora, Juiz de Fora, MG, Brasil \\ ${ }^{3}$ Departamento de Microbiologia, Imunologia e Parasitologia, Universidade Federal de Santa Catarina, Florianópolis, SC, Brasil
}

Four diamines and three amino alcohols derived from 1-decanol, 1-dodecanol and 1,2-dodecanediol were evaluated in an in vitro assay against a mixture of trypomastigote and intracellular amastigote forms of Trypanosoma cruzi. Two of these compounds (6 and 7) showed better activity against both proliferative stages of T. cruzi than the positive control benznidazole, three were of similar potency (1,2 and 5) and two were less active (3 and 4).

Key words: Chagas disease - Trypanosoma cruzi - amino alcohols - diamines - lipophilicity

Chagas disease is a neglected disease caused by the intracellular parasite Trypanosoma cruzi and affects mostly poor, rural and neglected populations, representing one of the main public health problems in the developing countries of Latin America (Clayton 2010). The current treatment for Chagas disease consists of nifurtimox and benznidazole, which were developed more than four decades ago (Coura \& de Castro 2002). However, these nitro compounds are considered far from ideal due to their multiple side effects and limited efficacy, particularly in patients suffering from the chronic form of Chagas disease (Rocha et al. 2007, Soeiro et al. 2009). These limitations highlight the urgent need for new trypanocidal compounds to replace the existing chemotherapy drugs.

We have shown in previous studies that lipophilic diamine and amino alcohol derivatives display inhibitory effects against Leishmania (da Costa et al. 2009), Trichomonas vaginalis (Giordani et al. 2009), Mycobacterium tuberculosis (de Almeida et al. 2007, Júnior et al. 2009) and on bloodstream trypomastigote forms of $T$. cruzi (Júnior et al. 2010). The aim of the present paper is a revaluation of the in vitro activity of these lipophilic diamines and amino alcohols in an assay consisting of L929 cells infected with both the amastigote and trypomastigote forms of the Tulahuen strain of T. cruzi. The trypomastigotes are the infective form of the parasite, whereas the amastigotes are the proliferating form, dividing for several generations before transforming into trypomastigotes and being released into the bloodstream. As the intracellular life cycle of $T$. cruzi can be repro-

doi: 10.1590/0074-0276130496

Financial support: CAPES, CNPq, FAPEMIG

+ Corresponding author: mauro.almeida@ufjf.edu.br

Received 10 October 2013

Accepted 3 December 2013 duced in cell culture, methodology using a multidose $T$. cruzi drug screening colourimetric assay allows for the rapid elimination of candidate compounds that produce no selective toxicity on the trypanosomes.

\section{MATERIALS AND METHODS}

In vitro assay using mixed trypomastigote and intracellular amastigote T. cruzi forms - The assay was performed according to the protocols established by Buckner et al. (1996), with reported modifications (Romanha et al. 2010). The $\beta$-galactosidase Tulahuen strain used was courtesy of Dr Frederick Buckner, University of Washington. Briefly, T. cruzi (Tulahuen strain) expressing the Escherichia coli $\beta$-galactosidase gene was grown on a monolayer of mouse L929 fibroblasts. Cultures assayed for $\beta$-galactosidase activity were grown in RPMI1640 medium without phenol red, plus $10 \%$ foetal bovine serum and glutamine. Tissue culture micro-plates (96 wells) were seeded with L929 fibroblasts at 4,000 cells per well in $80 \mu \mathrm{L}$ medium and incubated overnight at $37^{\circ} \mathrm{C}$ with $5 \% \mathrm{CO}_{2}$. $\beta$-galactosidase-expressing trypomastigotes were then added at 40,000 per well in a $20-\mu \mathrm{L}$ volume. After $2 \mathrm{~h}$ of contact, the medium containing trypomastigotes that had not penetrated the cells was discarded and replaced with $200 \mu \mathrm{L}$ of fresh medium. After $48 \mathrm{~h}$, the medium was discarded again and replaced with $180 \mu \mathrm{L}$ of fresh medium and $20 \mu \mathrm{L}$ of a solution of a test compound diluted with medium from stock solutions prepared using dimethyl sulfoxide; the final concentration was below $1 \%$, which showed no toxicity toward the parasite or L929 host cells (data not shown). Each compound was tested in triplicate at concentrations ranging from approximately $100-2 \mu \mathrm{M}$. After seven days of culture, chlorophenol red $\beta$-D-galactopyranoside at $100 \mu \mathrm{M}$ and Nonidet P-40 at $0.1 \%$ were added to the plates and incubated overnight at $37^{\circ} \mathrm{C}$. The absorbance was measured at $570 \mathrm{~nm}$ using an automated microplate reader. Benznidazole at its half maximal inhibitory concentration $\left(\mathrm{IC}_{50}\right)(1 \mu \mathrm{g} / \mathrm{mL}=3.84 \mu \mathrm{M})$ was used as a positive control. The results are expressed as a percentage of parasite inhibitory growth. 
Cytotoxicity assay - In vitro cytotoxicity tests were performed using alamarBlue ${ }^{\circledR}$ to determine the toxicity of the compounds against L929 cells. The conditions were the same as those described for the $\beta$-galactosidase assay. After $96 \mathrm{~h}$ of exposure to the compounds, alamarBlue ${ }^{\circledR}$ was added to each well and the absorbance at 570 and $600 \mathrm{~nm}$ was measured after 4-6 h. The lethal concentration $\left(\mathrm{LC}_{50}\right)$ and $\mathrm{IC}_{50}$ values were calculated using linear interpolation and the selectivity index (SI) was determined based on the ratio of the $\mathrm{LC}_{50}$ value for the host cell to the $\mathrm{IC}_{50}$ value for the parasite $\left(\mathrm{LC}_{50} / \mathrm{IC}_{50}\right.$ ratio) (Table). The activity and toxicity of benznidazole as the positive control were included for comparison.

\section{RESULTS AND DISCUSSION}

In a previous paper, we described the trypanocidal activity of lipophilic diamines and amino alcohols on bloodstream trypomastigotes, with all the tested compounds displaying an activity ranging from $\mathrm{IC}_{50}$ values of 147 to $1,020 \mu \mathrm{M}$ (Júnior et al. 2010). In the present work, the activity of the same compounds was assayed using a tissue cell culture system, which is considered the in vitro method of choice for screening drugs for activity against T. cruzi (Canavaci et al. 2010, Romanha et al. 2010).
The results showed that the tested compounds displayed higher activities against the T. cruzi culture form ( $\mathrm{IC}_{50}$ values ranging from 2.46-18.31 $\mu \mathrm{M}$ ) (Table), being at least 10 -fold more active compared to the previous data observed for the bloodstream form (Júnior et al. 2010). A greater sensitivity of the amastigote form of $T$. cruzi has also been observed by other researchers using in vitro assays (da Silva et al. 2010). The in vitro activity of two of these compounds (6 and 7) against both proliferative stages of $T$. cruzi was superior to the positive control benznidazole (Table); in contrast, three exhibited similar potencies (1,2 and 5) and two were substantially less potent (3 and 4). These results suggest a greater effectiveness of these compounds on intracellular parasites and different capabilities in drug uptake and/or extrusion mechanisms could explain the greater sensitivity of the amastigotes to the tested compounds compared to the bloodstream trypomastigotes. However, to the best of our knowledge, there is no study showing a difference between the amastigote and trypomastigote forms relative to uptake and extrusion mechanisms. A second hypothesis is that the compounds could have different targets of amastigote and trypomastigote forms. A third hypothesis is that the mimicry of blood bank conditions,

TABLE

Trypanocidal activity, cytotoxicity and selectivity index (SI) of lipophilic diamines and amino alcohols in mixed trypomastigote and amastigote culture of Trypanosoma cruzi

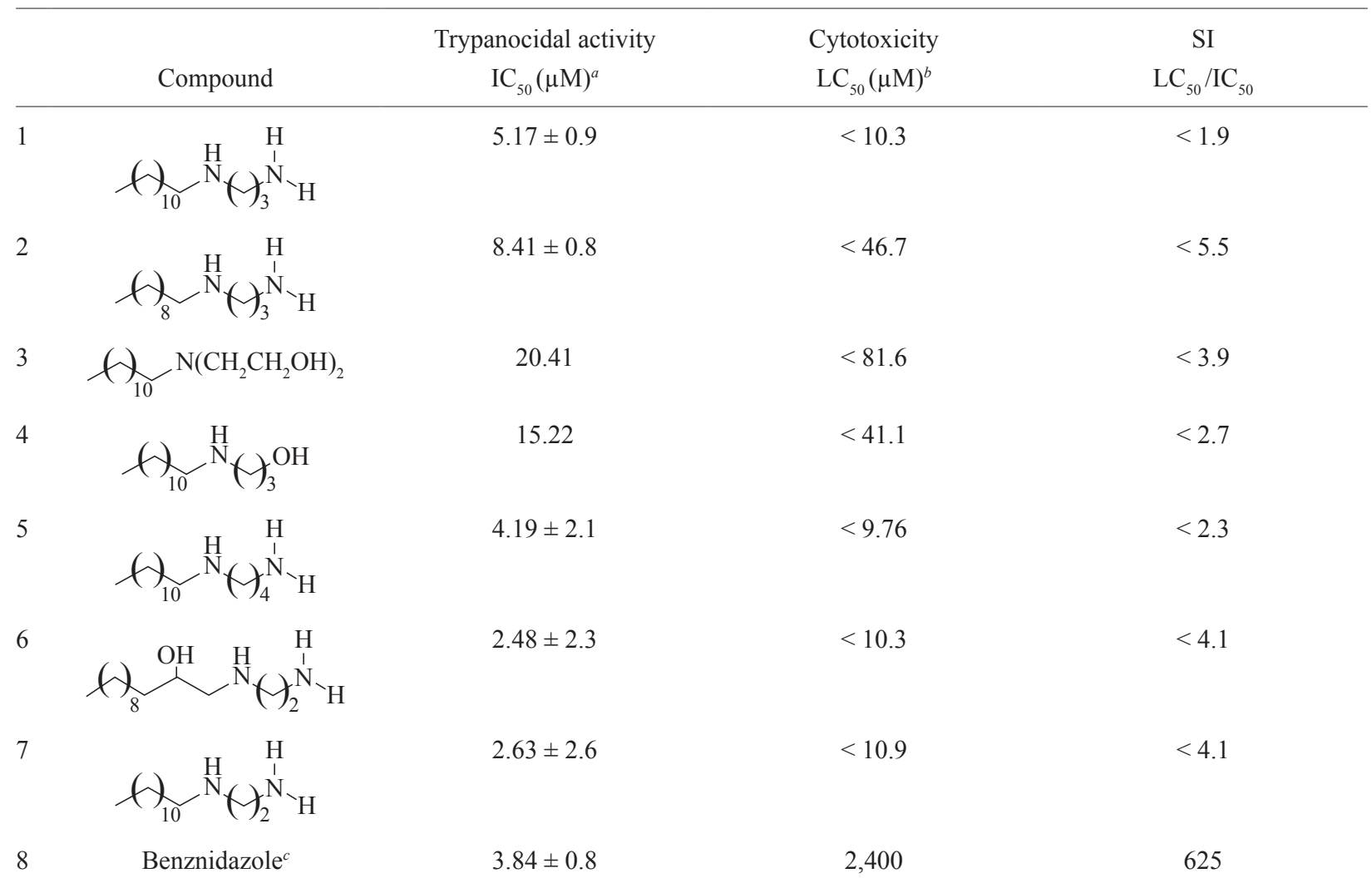

$a$ : compound $\mu$ molar concentration that inhibits $50 \%$ of the growth of the culture of mixed amastigote and trypomastigote forms of T. cruzi; $b$ : compound $\mu$ molar concentration that reduces $50 \%$ of the L929 cell viability. Except for compounds 3 and 4 (once), trypanocidal and cytotoxicity assays were performed twice; $c$ : positive control; IC: inhibitory concentration; LC: lethal concentration. 
i.e., short assay incubation time ( $24 \mathrm{~h})$, low temperature $\left(4^{\circ} \mathrm{C}\right)$ and inactivation by serum components, contributed to the lower trypanocidal activity over the bloodstream trypomastigotes. Lastly, the parasite strain could explain these results: a partially drug-resistant strain (Y) in the previous work vs. a drug-susceptible strain (Tulahuen) in the present study. In fact, there is a broad spectrum of bioactivity of different drugs against different strains of $T$. cruzi (Filardi \& Brener 1987), which emphasises the need to analyse the activity of new compounds against different strains, including strains susceptible, partially resistant and resistant to benznidazole and nifurtimox.

Thus, aiming to determine the dose toxic to uninfected L929 host cells, we used the same cell number, time of cell development and time of compound exposure used for the $\beta$-galactosidase assay. The cell viability was determined at $96 \mathrm{~h}$ of incubation with different concentrations of each compound. The compounds induced $100 \%$ cell death at doses raging from 9.76-73.27 $\mu \mathrm{M}$ (Table). The SI reflects the extent to which each compound is active against the parasite without inducing toxicity toward the host cell. The SI value of each compound was determined based on the $\mathrm{IC}_{50}$ for the parasites and $\mathrm{LC}_{50}$ for the $\mathrm{L} 929$ cells and ranged from $<1.9$ to $<5.5$ (Table).

The results described in this paper together with the results described by Júnior et al. (2010) suggest that lipophilic diamine and amino alcohol compounds could be prototypes for new trypanocidal drugs, as they showed trypanocidal activity over different Trypanosoma culture forms and parasite strains and also under different assay conditions. According to Romanha et al. (2010), a promising compound for the treatment of Chagas disease must (i) have activity against bloodstream trypomastigote and amastigote forms, (ii) act upon a large panel of parasite isolates, including those that express natural resistance to benznidazole and nifurtimox, (iii) show similar or greater trypanocidal effects than the reference drug benznidazole and (iv) show a selective index equal or greater than 50 ( $\mathrm{SI} \geq 50$ ). Unfortunately, the fatty diamines and amino alcohols do not fulfil all these parameters. Indeed, based on the decision criteria of Romanha et al. (2010) for the determination of the efficacy of novel drugs against $T$. cruzi, the compounds would not be considered for in vivo tests because the therapeutic index is too low $(\mathrm{SI}<50)$.

\section{ACKNOWLEDGEMENTS}

To PDTIS-FIOCRUZ, for use of its facilities.

\section{REFERENCES}

Buckner F, Verlinde CLMJ, la Flamme AC, van Voorhis WC 1996. Efficient technique for screening drugs for activity against Trypanosoma cruzi using parasites expressing galactosidase. Antimicrob Agents Chemother 40: 2592-2597.

Canavaci AMC, Bustamante JM, Padilla AM, Brandan CMP, Simpson LJ, Xu D, Boehlke CL, Tarleton RL 2010. In vitro and in vivo high-throughput assays for the testing of anti-Trypanosoma cruzi compounds. PLoS Negl Trop Dis 4: e740.

Clayton J 2010. Chagas disease: pushing through the pipeline. Nature 465 (Suppl.): S12-S15.

Coura JR, de Castro SL 2002. A critical review on Chagas disease chemotherapy. Mem Inst Oswaldo Cruz 97: 3-24.

da Costa CF, Coimbra ES, Braga FG, dos Reis RCN, da Silva AD, de Almeida MV 2009. Preparation and antileishmanial activity of lipophilic $N$-alkyl diamines. Biomed Pharmacother 63: 40-42.

da Silva CF, da Silva PB, Batista MM, Daliry A, Tidwell RR, Soeiro MNC 2010. The biological in vitro effect and selectivity of aromatic dicationic compounds on Trypanosoma cruzi. Mem Inst Oswaldo Cruz 105: 239-245.

de Almeida MV, le Hyaric M, Amarante GW, Lourenço MCS, Brandão MLL 2007. Synthesis of amphiphilic galactopyranosyl diamines and amino alcohols as antitubercular agents. Eur J Med Chem 42: 1076-1083.

Filardi LS, Brener Z 1987. Susceptibility and natural resistance of Trypanosoma cruzi strains to drugs used clinically in Chagas disease. Trans R Soc Trop Med Hyg 81: 755-759.

Giordani RB, de Almeida MV, Fernandes E, da Costa CF, de Carli GA, Tasca T, Zuanazzi JAS 2009. Anti-Trichomonas vaginalis activity of synthetic lipophilic diamine and amino alcohol derivatives. Biomed Pharmacother 63: 613-617.

Júnior COR, Alves RO, Rezende CAM, da Costa CF, Silva H, le Hyaric M, Fontes APS, Alves RJ, Romanha AJ, de Almeida MV 2010. Trypanocidal activity of lipophilic diamines and amino alcohols. Biomed Pharmacother 64: 624-626.

Júnior COR, le Hyaric M, da Costa CF, Corrêa TA, Taveira AF, Araújo DP, Reis EFC, Lourenço MCS, Vicente FRC, de Almeida MV 2009. Preparation and antitubercular activity of lipophilic diamines and amino alcohols. Mem Inst Oswaldo Cruz 104: 703-705.

Rocha MO, Teixeira MM, Ribeiro AL 2007. An update on the management of Chagas cardiomyopathy. Expert Rev Anti Infect Ther 5: 727-743.

Romanha AJ, de Castro SL, Soeiro MNC, Lannes-Vieira J, Ribeiro I, Talvani A, Bourdin B, Blum B, Olivieri B, Zani C, Spadafora C, Chiari E, Chatelain E, Chaves G, Calzada JE, Bustamante JM, Freitas-Junior LH, Romero LI, Bahia MT, Lotrowska M, Soares M, Andrade SG, Armstrong T, Degrave W, Andrade ZA 2010. In vitro and in vivo experimental models for drug screening and development for Chagas disease. Mem Inst Oswaldo Cruz 105: 233-238.

Soeiro MNC, Dantas AP, Daliry A, da Silva CF, Batista DGJ, de Souza EM, Oliveira GM, Salomão K, Batista MM, Pacheco MGO, da Silva PB, Santa-Rita RM, Barreto RFSM, Boykin DW, de Castro SL 2009. Experimental chemotherapy for Chagas disease: 15 years of research contributions from in vivo and in vitro studies. Mem Inst Oswaldo Cruz 104 (Suppl. I): 301-310. 